\title{
The application of Picard method in identification of the heat transfer coefficient in flow boiling in a minichannel
}

\author{
Mirosław Grabowski ${ }^{1}$, Sylwia Hożejowska ${ }^{2, *}$, Anna Pawińska $^{2}$, and Mieczysław E. Poniewski ${ }^{1}$ \\ ${ }^{1}$ Warsaw University of Technology, Płock Campus, ul. Łukasiewicza 17, 09-400 Płock, Poland \\ ${ }^{2}$ Kielce University of Technology, Faculty of Management and Computer Modelling, Al.1000-lecia PP 7, 25-314 Kielce, Poland
}

\begin{abstract}
This paper summarizes the procedure of experimental determination of flow boiling heat transfer coefficient in the rectangular minichannel $2 \mathrm{~mm}$ wide, $1.8 \mathrm{~mm}$ deep and $193 \mathrm{~mm}$ long. In this experiment, the basic thermal and flow parameters were measured and the temperature distribution on the foil insulating the heater was recorded using an infrared camera. A high speed video camera observed the vapor patterns forming during the boiling process. The void fraction was determined with the image processing technique. Stationary heat transfer process in the insulating foil, the heater and the flowing liquid was described using two-dimensional Laplace's equation (for the foil), the Poisson equation (for the heater) and energy equation (for the liquid) - all complemented with an appropriate system of boundary conditions. The system of the differential equations with adopted boundary conditions gives the three consecutive inverse problems in elements of the test section containing minichanel. Trefftz method was used to determine two dimensional temperature distribution of the foil and the heater. The liquid temperature was calculated using the PicardTrefftz method. The values of the experimentally obtained heat transfer coefficients with the values of these coefficients calculated on the basis of correlations known from the literature were also compared.
\end{abstract}

\section{Introduction}

Recent years have been abundant with experimental and theoretical works on thermal processes occurring in flow boiling in mini- and micro channels. A wide and comprehensive review of the accomplishments in the field is presented in [1], where much has been said about mathematical models and their application to mathematical analysis. The most general model, that is, two-fluid four-field model that at least theoretically allows continuous modelling of flow changes, has been discussed in [2]. This model comprises a system of several partial differential equations, even when related to developed nucleate boiling. Additionally, a suitable set of boundary conditions has to be defined. Solving such a complex system of equations is not always possible, especially in the case of inverse problems. Even commercial CFD software may be inefficient in such a case. Therefore, there is a need to develop numerical and other methods for solving inverse problems especially coupled inverse problems in areas in contact, with different physical parameters.

The methods presented here: 1) the Trefftz method and 2) the Picard -Trefftz method, belong to the class of semianalytical methods for solving partial differential equations. The Trefftz method was first described by Trefftz [3] and was addressed to linear equations. This method was generalized by many authors [4-8] who expanded its applicability. The paper presents application of the Trefftz method and the Picard-Trefftz method to an approximate solution of the inverse heat conduction problems for stationary flow of a refrigerant in rectangular and horizontal minichannel. The temperature distribution of the elements of the minichannel insulating foil, heating foil and boiling liquid was described with the Laplace equation, Poisson equation and stationary energy equation, respectively. The Trefftz method was used to obtain two dimensional temperature distributions of the insulating foil and of the heater. Two dimensional fluid temperatures was calculated with the use of the Picard - Trefftz method. The heat transfer coefficient at the heater-liquid interface was determined based on the boundary condition of the third kind. Local values of the experimental heat transfer coefficient were compared with the values obtained with correlations of developed experimentally by other researchers.

\section{Experiment}

\subsection{Experimental stand}

Figure 1 shows the flow boiling apparatus used. Its main part is the measuring section, Fig.1 \#1. Containing the milled in the body, a $193 \mathrm{~mm}$ long minichannel with a rectangular cross-section ( $2 \mathrm{~mm}$ wide, $1.8 \mathrm{~mm}$ deep). A $2 \mathrm{~mm}$ thick transparent lid with a sodium glass visor closes the minichannel on one side, and a heater in the

* Corresponding author: ztpsf@tu.kielce.pl 
form of a flat strip made of Fecral 135 retaining alloy with dimensions of $2 \times 0.1 \mathrm{~mm}$, sealed with insulating foil (3M SCOTCH S 33+ insulating tape) is mounted on the other side. Czaki TP-201 type K thermocouples, $0.5 \mathrm{~mm}$ in diameter, and Kobold pressure sensors with a range of $0-2.5$ bar (accuracy $\pm 1 \%$ ) were also mounted in the body. The both sensor types are shown schematically in Fig.1, \#P and \#T. The heater is powered from the programmable DC power supply by TDK Lambda, Fig.1 \#2.

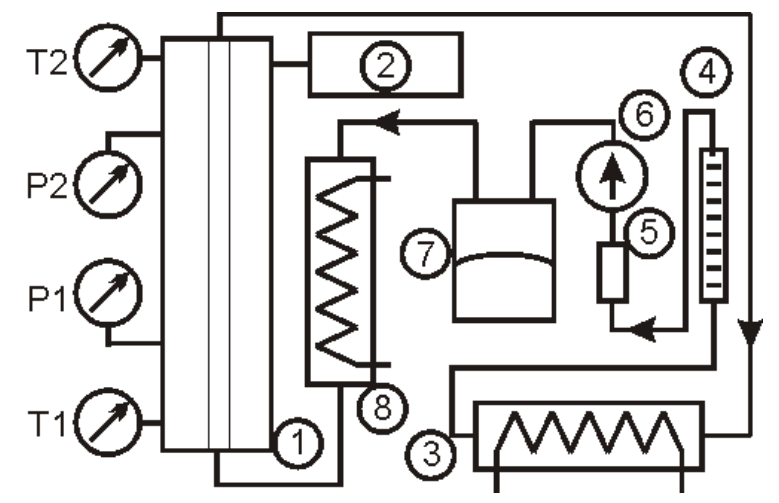

Fig.1. Flow loop: 1 - test section with the minichannel (described in detail in Figure 2), 2 - DC power supply for the heater, 3 - cooler, 4 - rotameter, 5 - filter, 6 - pump, 7 - pressure control, 8 - preheater.

Vapor structures forming due to a phase change during the liquid heating are observed and recorded using a Vision Research Phantom 710 high speed camera with a resolution of $1280 \times 800$ pixels at 7530 frames per second.

The two-dimensional temperature distribution on the external surface of the heater is measured and recorded using the FLIR SC 7600 Series infrared camera with a recording resolution of 640x512 pixels and recording frequency of up to $100 \mathrm{~Hz}$.

The experimental set-up was designed to:

- displace/force the test liquid at a given temperature (Fig.1, heater \#8) and a given pressure through the flow loop (Fig.1, pump \#6, pressure set $\# 7$ ), with a maximum volumetric flow rate up to $1.5 \cdot 10^{-7} \mathrm{~m}^{3} \mathrm{~s}^{-1}$ (Fig.1, rotameter \#4);

- preheat the liquid flowing into the minichannel using the preheater (Fig.1 \#8), and then overheat it above the saturation temperature in the minichannel located in the measurement section (Fig.1 \#1) using the heater (not visible in the diagram);

- measure and record the following parameters: liquid pressure and temperature at the inlet and outlet of the minichannel, Fig.1a, manometers P and thermocouples $\mathrm{T}$;
- volumetric flow rate of the tested liquid, Fig.1, rotameter \#4;

- thermal power supplied to the heating foil, Fig.1, current and voltage supplied by the DC power supply \#2;

- concurrently and thermographically visualize the heating foil temperature distribution and provide images of two-phase flow patterns.

\subsection{Experimental results}

The experimental setup described above and in [9] enabled to collect the folowing parameters:the flow of the liquid at a set rate, temperature and pressure at the inlet to the minichannel, to heat the liquid in the minichannel to the set temperature, to measure and record the temperature and pressure at the inlet and outlet of the minichannel, liquid volumetric flow rate, and the electric current supplied to the heating foil, and to record the flow structures and two-dimensional temperature distributions on the outer surface of the insulation foil.

The temperature distributions recorded with the thermal imaging camera on the insulating foil were particularly important for further calculations, Fig. 2.

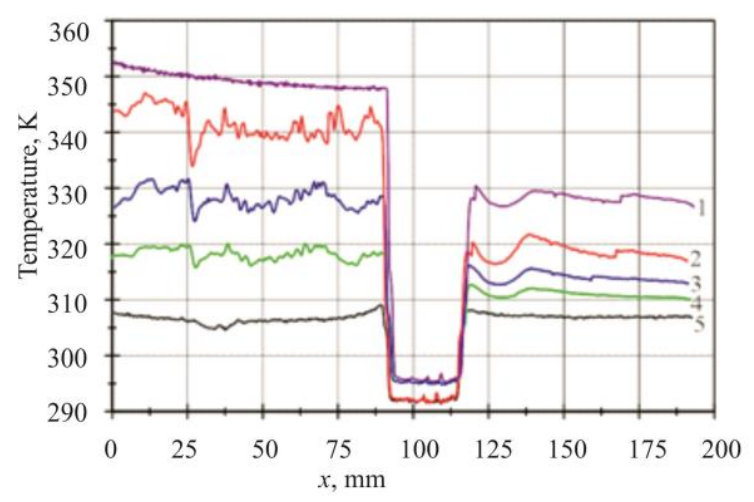

Fig. 2. Exemplary temperature profiles on the minichannel side in contact with ambient surroundings as a function of the distance from the channel inlet. Volumetric flow rate for all cases was $1.41 \cdot 10^{-7} \mathrm{~m}^{3} / \mathrm{s}$. Case 1: heat flux $154086 \mathrm{~W} / \mathrm{m}^{2}$, Case 2: heat flux $102634 \mathrm{~W} / \mathrm{m}^{2}$, Case 3: heat flux 55699W/m², Case 4: heat flux $24194 \mathrm{~W} / \mathrm{m}^{2}$, Case 5: heat flux $0 \mathrm{~W} / \mathrm{m}^{2}$.

They formed the basis for determining heat transfer coefficients in the minichannel using the Picard-Trefftz method. Observations of the two-phase flow structures helped determine the void fraction, The method of determining the void faction was described in detail in [10].

The minichannel was split into three domains related to the heat supply method used. In the domain $\Omega_{\mathrm{I}}$, the heat supplied to the liquid was generated by the electric heater. Due to design reasons, it was not possible to record the temperature field in the foil insulating of the 
domain $\Omega_{\text {III }}$. The domain $\Omega_{\text {III }}$ was quasi-adiabatic and all thermal and flow parameters were recorded there, just as in $\Omega_{\text {I }}$, Fig 3 .

\section{Mathematical model and methods}

The presented mathematical model is a modification of the approaches from $[9,11]$. Two dimensions were taken into account in the mathematical model: one in the direction of the flow $(x)$ and one in the perpendicular direction $(y)$ referred to the foil thickness $\left(\delta_{F}\right)$, to the heater $\left(\delta_{H}\right)$ and to the depth of the minichannel $\left(\delta_{M}\right)$. Assumption that the phenomena occurring at the side surfaces of the measurement module do not affect the thermodynamic parameters of its central part allows limiting the considerations to this central part only (along its length $L$ ).

Due to the measurement module design this mathematical model concerns heat transfer in only first $\Omega_{\mathrm{I}}$ region where in the section with a length of $L_{\mathrm{I}}=93 \mathrm{~mm}$ there is the heater heating the liquid flowing into the minichannel.

It was assumed that the insulating foil temperature $T_{F}$ and the heater temperature $T_{H}$ satisfy the Laplace and Poisson equations in the form, $[9,11]$

$$
\begin{aligned}
& \Delta T_{F}=0 \text { for } 0<x<L_{1}, 0<y<\delta_{F} \\
& \Delta T_{H}=-\frac{q_{V}}{\lambda_{H}} \text { for } 0<x<L_{1}, \delta_{F}<y<\delta_{F}+\delta_{H}
\end{aligned}
$$

where $\Delta=\frac{\partial^{2}}{\partial x^{2}}+\frac{\partial^{2}}{\partial y^{2}}, L_{\mathrm{I}}-$ length of the domain $\Omega_{\mathrm{I}}$, $q_{V}$ - volumetric heat flux supplied to the heater, $\lambda_{H}-$ the thermal conductivity of the heater. For equations (1) and (2) the assumption was that:

- at the foil-heater interface the temperature and heat fluxes were equal,

- at the foil - external environment contact the temperature of the foil was equal to the temperature measurements (conducted by infrared thermography) and

$$
\lambda_{F} \frac{\partial T_{F}}{\partial y}=-q_{\text {loss }} \text { for } y=0,0 \leq x \leq L_{1}
$$

where $q_{\text {loss }}$ - the heat loss to the surroundings was determined as in [12],

- the remaining boundaries are isolated, Fig. 3 .

It was assumed that the fluid flow in the minichannel was steady state and laminar (Reynolds number $R e<2000$ ) with a constant volumetric flow rate, [11]. Additionally, the fluid velocity in the minichannel has only one non-zero component $u(y)$ with parabolic profile, parallel to the flow direction and determined by analogy with [11].

The fluid temperature $T_{f}$ in the domain $\Omega_{\mathrm{I}, f}$ satisfied the energy equation

$$
\lambda_{f} \Delta T_{f}-u(y) c_{p} \rho_{f} \frac{\partial T_{f}}{\partial x}=0
$$

where $\lambda_{f}-$ the thermal conductivity of the fluid, $c_{p}$-specific heat, $\rho_{f}-$ fluid density, $u(y)-$ liquid velocity.

Adequate boundary conditions were adopted for equation (4), Fig. 3:

$$
\begin{array}{r}
T_{f}\left(x, \delta_{F}+\delta_{H}\right)=T_{H}\left(x, \delta_{F}+\delta_{H}\right) \text { for } 0 \leq x \leq L_{\mathrm{I}} \\
T_{f}(0, y)=T_{\text {in }} \text { for } \delta_{F}+\delta_{H} \leq y \leq \delta_{F}+\delta_{H}+\delta_{M} \\
T_{f}\left(L_{\mathrm{I}}, y\right)=T_{s a t}\left(L_{\mathrm{I}}\right) \text { for } \delta_{F}+\delta_{H} \leq y \leq \delta_{F}+\delta_{H}+\delta_{M}
\end{array}
$$

where $T_{i n}$ - the liquid temperature at the minichannel inlet, $T_{\text {sat }}$-the saturation temperature depends on linearly changing pressure.

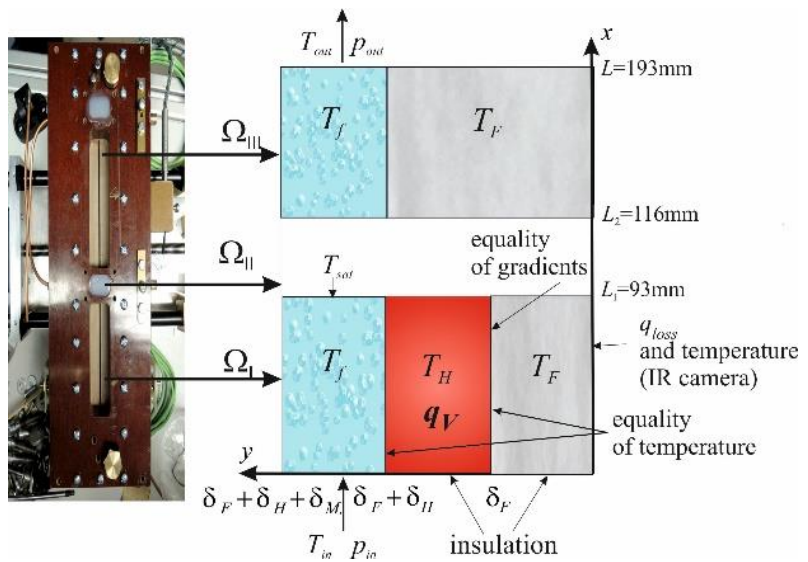

Fig. 3. Test section and its schematic illustrating the underlying model assumptions (pictorial view, not to scale).

For the considered bubbly and bubbly-slug flow the heat flux generated in the heater is transferred to the liquid phase in the proportion relative to the void fraction $\varphi(x)$. Therefore, the heat transfer coefficient at the foil-liquid interface was determined using the Robin condition in the form

$$
\alpha_{2 D}(x)=(1-\varphi(x)) \frac{-\lambda_{H} \frac{\partial T_{H}}{\partial y}\left(x, \delta_{F}+\delta_{H}\right)}{T_{H}\left(x, \delta_{F}+\delta_{H}\right)-T_{a v e}(x)}
$$

where the reference temperature $T_{\text {ave }}$ was the average fluid temperature along the minichannel depth

$$
T_{\text {ave }}(x)=\frac{1}{0.5 \delta_{M}} \int_{\delta_{F}+\delta_{H}}^{\delta_{F}+\delta_{H}+0.5 \delta_{M}} T_{f}(x, y) d y
$$

The main idea of the Picard-Trefftz method is a decomposition of the differential operator from equation (4) into the Laplace operator and the remaining component of equation (4). Next, the Picard's iterations were used where in each iteration a linear combination of the Trefftz functions for the Laplace operator was used, [13] 
Equation (4) with the conditions (5) - (7) can be written using operator notation

$$
\begin{aligned}
& \Delta T_{f}+N T_{f}=0 \text { for }(x, y) \in \Omega_{\mathrm{I}, f} \\
& B T_{f}=g(x, y) \text { for }(x, y) \in \partial \Omega_{\mathrm{I}, f}
\end{aligned}
$$

where differential operator $N$ is defined as

$$
N=-u(y) \frac{c_{p} \rho_{f}}{\lambda_{f}} \frac{\partial}{\partial x}
$$

and operator $B$ describes boundary conditions (5)- (7).

In the Picard iterations the following procedure was used to approximate the solutions to equation (4):

- in the first step, for $k=1$ :

$$
\begin{gathered}
\Delta T_{f}^{(1)}=0 \text { for }(x, y) \in \Omega_{\mathrm{I}, f} \\
B T_{f}^{(1)}=g(x, y) \text { for }(x, y) \in \partial \Omega_{\mathrm{I}, f}
\end{gathered}
$$

- in subsequent steps, for $k>1$ :

$$
\begin{aligned}
& \Delta T_{f}^{(k)}=-N T_{f}^{(k-1)} \text { for }(x, y) \in \Omega_{\mathrm{I}, f} \\
& B T_{f}^{(k)}=g(x, y) \text { for }(x, y) \in \partial \Omega_{\mathrm{I}, f}
\end{aligned}
$$

In each step the Trefftz method was used to determine the approximate solutions to equations (13) and (15) as in [13]. The unknown solutions equations (13) and (15) was approximating in the form of a linear combination of the Trefftz functions $w_{j}(x, y)$ for Laplace equation, i.e.

$$
T_{f}^{(k)}=\sum_{j=1}^{M^{(k)}} a_{j}^{(k)} w_{j}(x, y)+T_{s o l}^{(k)}(x, y)
$$

where $T_{\text {sol }}^{(k)}(x, y)$ is a particular solution to the Poisson's equation (15) and is given by formula

$$
T_{\text {sol }}^{(k)}=-\Delta^{-1}\left[N T_{f}^{(k-1)}\right]
$$

and the particular solution equation (13) is equal to zero, $T_{\text {sol }}^{(1)}(x, y)=0$. In (18) operator $\Delta^{-1}$ is calculated according to the formula

$$
\Delta^{-1}\left(x^{m} y^{l}\right)=m ! l ! \sum_{k=0}^{[m} \sum^{2]}(-1)^{k} \frac{x^{m-2 k} y^{l+2+2 k}}{(m-2 k) !(l+2+2 k) !}
$$

where the symbol [.] denotes the floor function.

The coefficients of the linear combinations in (17) were determined based on the adopted boundary conditions, following the method described in [14].

\section{Results and discussion}

Numerical calculations were performed using the Trefftz method and the Picard -Trefftz method for the twophase flow of ethanol in a $1.8 \mathrm{~mm}$ deep and $2.0 \mathrm{~mm}$ wide horizontal minichannel. The following experimental data were used in calculations: inlet pressure $10.9 \mathrm{kPa}$ (average), liquid temperature at the inlet $306 \mathrm{~K}$ (average), volume flow rate $8.2 \cdot 10^{-8} \mathrm{~m}^{3} \mathrm{~s}^{-1}$ and five heat flux densities $q_{w}$ in the range of $99.2 \div$ $154.1 \mathrm{kWm}^{-2}$. The thermal imaging camera helped to measure the temperature distribution of the insulating foil. In further calculations, the local void fraction determined, in the manner described in [10], at the distances of $0 \mathrm{~m}, 0.02 \mathrm{~m}, 0.04 \mathrm{~m}, 0.06 \mathrm{~m}$, and $0.08 \mathrm{~m}$ from the minichannel inlet was approximated with a quadratic function, Fig. 4.

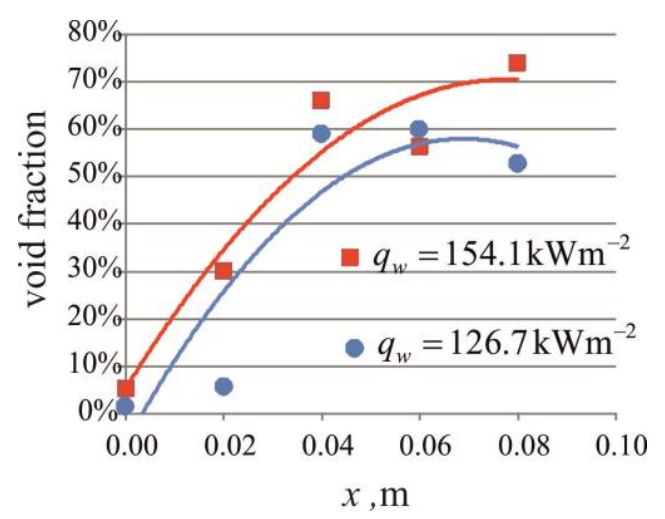

Fig. 4. The void fraction approximated by quadratic function.

As follows from the presented mathematical approach two-dimensional temperature distributions in the insulating foil and the heater were determined first using the Trefftz method with six harmonic polynomials (Trefftz functions for Laplace equation). In the next stage, the liquid temperature distribution in the minichannel was determined by the Picard-Trefftz method with four iterative steps and two harmonic polynomials at each step.

The values of local heat transfer coefficients obtained from (8) are shown in Fig. 5. It was observed that when the heat flux supplied to heating foil grows, the fraction of the vapour phase increases, leading to the decrease of the heat transfer coefficient.

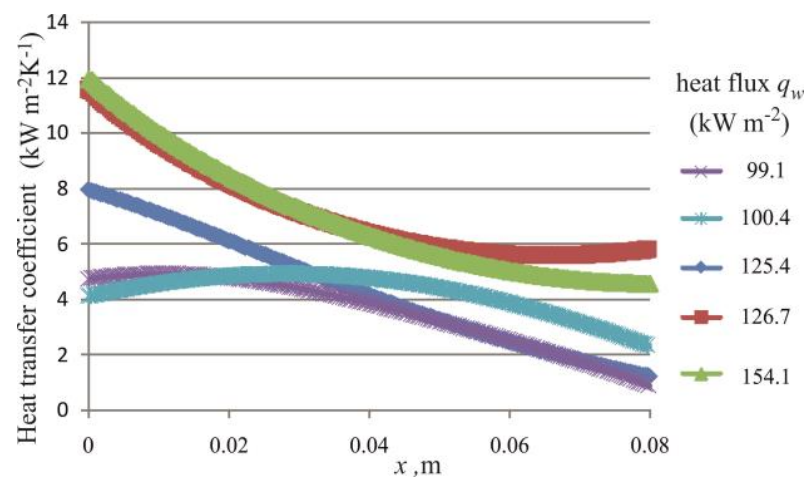

Fig. 5. Heat transfer coefficients for different heat fluxes as a function of the minichannel length obtained using the Picard-Trefftz method.

This paper compared the values of the experimental heat transfer coefficient $\alpha_{2 D}$ calculated from formula (8) with the values $\alpha_{T P}$ calculated based correlations known from the literature: 
-Kandlikar and Balasubramanian correlation for low Reynolds numbers [15]:

$$
\alpha_{T P}=\left(0.6683 \cdot C o^{-0.2}+B o^{0.7} F_{F l}\right)(1-X)^{0.8} \alpha_{S P}
$$

where fluid-surface parameter $F_{F l}$ and the heat transfer coefficient $\alpha_{S P}$ for a single phase flow were determined as in [15]:

-Bohdal and Charun [16]:

$$
\alpha_{T P}=19.2 \operatorname{Re}^{0.8} B o^{0.6}\left(\frac{\rho_{g}}{\rho_{l}}\right)^{0.5} \frac{\lambda_{f}}{D}
$$

- Gungor and Winterton [17]:

$$
\begin{gathered}
\alpha_{T P}=E \cdot 0.023 \operatorname{Re}_{l}^{0.8} \operatorname{Pr}_{l}^{0.4} \frac{\lambda_{f}}{D} \\
E=1+3000 B o^{0.86}+1.12\left(\frac{X}{1-X}\right)^{0.75}\left(\frac{\rho_{l}}{\rho_{g}}\right)^{0.41}
\end{gathered}
$$

- Tran and Wambsganss [18]:

$$
\alpha_{T P}=840000\left(B o^{2} W e_{l}\right)^{0.3}\left(\frac{\rho_{g}}{\rho_{l}}\right)^{0.4}
$$

The following symbols were adopted in correlations (20) - (23) the following symbols means: Bo-boiling number, $C o$-convection number, $X$-vapour quality, $D$-minichannel internal perimeter, $P r$ - Prandtl number, $R e$-Reynolds number, $W e-$ Weber number, subscripts $l$ and $g$ refers to the liquid and gas phase respectively.

Figure 6 compares the values of heat transfer coefficient calculated based on correlations (20) - (23) and the values of experimental coefficient $\alpha_{2 D}$ calculated from (8) for $q_{w}=125.2 \mathrm{kWm}^{-2}$.

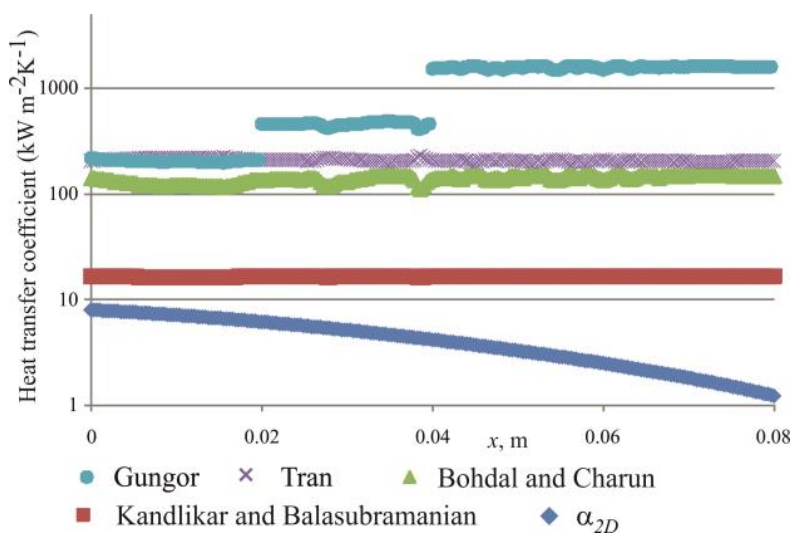

Fig.6. Local heat transfer coefficients calculated based on correlations (20)-(23) and formula (11) for $q_{w}=125.2 \mathrm{kWm}^{-2}$.

\section{Conclusions}

The proposed 2D stationary mathematical approach was constructed for the heat transfer in three adjacent elements of the test section. Trefftz method was used to determine two dimensional temperature distribution of the insulating foil and the heater. The liquid temperature was calculated using the Picard-Trefftz method. The heat transfer coefficients obtained from the two-dimensional approach were compared with correlations known from the literature.

The heat transfer coefficients calculated from the Kandlikar and Balasubramanian correlations [15] were most similar to those calculated experimentally from (8), Fig.5. With other correlations the differences between the experimental and correlation-based results were more pronounced. These considerable differences may result from the fact that the correlations in question have been developed for the flows with higher Reynolds numbers than those in our experiment. From the descriptions of experiments performed by the cited researchers, it appears that heat losses to the environment are often omitted, with a comment added that they can have significant value. This inaccuracy in determining the heat flux transmitted to the fluid may also explain obtaining by the cited authors coefficient values consistently higher compared to those presented in these study, where heat losses are included in the calculation procedure.

This opens a new field of research devoted to the modification of the existing correlations or the development of original correlation equation.

\section{References}

1. Z. Guo, D.F. Fletcher, B.S. Haynes, J. Comput. Multiphase Flows 6 (2014)

2. R.T. Lahey Jr., Proc. XIII US National Congress on Applied Mechanics, June 21-26, University of Florida, USA (1998)

3. E. Trefftz, Proc. II Int.Congress of Applied Mechanics, Zurich, 131-137 (1926)

4. O.C. Zienkiewicz, D.W. Kelly, P. Bettes, Int. J. Numer. Meth. Eng. 11 (1977)

5. J. Jirousek 1978, Comput. Meth. App. Mech. Eng. 14 (1978)

6. I. Herrera, Boundary methods. An algebraic theory, (Pitman, Boston-London-Melbourne, 1984)

7. Z.-C. Li, T.-T. Lu, H.-Y. Hu, A. H.-D. Cheng., Trefftz and Collocation Methods, (WIT Press, Southampton 2008)

8. K. Grysa, A. Maciąg, Int. J. Heat Mass Transfer 100, (2016)

9. S. Hożejowska, M. Grabowski, EPJ Web of Conf. 114, 02041 (2016)

10. K. Płaczkowski, M. Poniewski, M. Grabowski, S. Alabrudziński, App. Mech. Materials 797 (2015)

11. S. Hożejowska, R. Kaniowski, M.E. Poniewski, Exp. Thermal Fluid Sci.78 (2016)

12. M. Piasecka, K. Strąk, B. Maciejewska, Heat Transfer Eng. 38 (2016)

13. K. Grysa, A. Maciąg, A. Pawińska, Int. J. Heat Mass Transfer 55 (2012) 
14. S. Hożejowska, M. Piasecka, M. Poniewski, Int. J. Therm. Sci. 48 (2009)

15. S.G. Kandlikar, P. Balasubramanian, Heat Transfer Eng. 25, 3 (2004)

16. T. Bohdal, H. Charun, K.Dutkowski, 2008, Annals of Environment Protection 10 (2008)

17. K.E. Gungor, R.H.S. Winterton, Chem. Eng. Res. Des. 65 (1987)

18. T. Tran, M.W. Wambsganss, D.M. France, Int. J. Multiphase Flow 22 (1996) 